\title{
Diffraction coupled phase-locked semiconductor laser array
}

\author{
J. Katz \\ Jet Propulsion Laboratory, California Institute of Technology, Pasadena, California 91109 \\ S. Margalit and A. Yariv \\ Department of Electrical Engineering, California Institute of Technology, Pasadena, California 91125
}

(Received 6 December 1982; accepted for publication 10 January 1983)

\begin{abstract}
A new monolithic, diffraction coupled phase-locked semiconductor laser array has been fabricated. Stable narrow far-field patterns $\left(\sim 3^{\circ}\right)$ and peak power levels of $1 \mathrm{~W}$ have been obtained for $100-\mu \mathrm{m}$-wide devices with threshold currents as low as $250 \mathrm{~mA}$. Such devices may be useful in applications where high power levels and stable radiation patterns are needed.
\end{abstract}

PACS numbers: 42.60. By

Many applications of semiconductor injection lasers can benefit from achieving higher levels of emitted power than those available from a single device in a stable radiation pattern. A possible solution to this problem is to coherently combine the power of several lasers operating on the same substrate. This approach has been the subject of an intense research effort in recent years. ${ }^{1-12}$ In these works, phase locking of the lasers is achieved via distributed coupling among the lasers of the array along their intrinsic waveguides, as was demonstrated by Ref. 13. Using this method, the resulting farfield in may cases has basically a double-lobe pattern $^{4-6,8,10,12}$ because the fields of adjacent lasers are in antiphase to each other. Generally, the phase between adjacent lasers depends upon the particular lasers used in the array. Recently, Streifer and coworkers have obtained arrays with excellent performance parameters in terms of emitted power ${ }^{10}$ and an extremely narrow single-lobe farfield pattern. ${ }^{11}$

In this letter we report on another approach for monolithic implementation of a phase-locked laser array which is based on diffraction coupling. In this configuration, shown in Fig. 1, each laser is uncoupled from its neighbors along a part of the total resonator length, but all the lasers share a common section of the resonator in the region near the mirrors. Light from the uncoupled (waveguide) section of the laser propagates in the common resonator section where it diffracts. Upon reflection from the resonator mirror, some of the light is reflected back into the waveguide section, as is the case in any laser. However, fractions of this field are also reflected back into the adjacent lasers, thus constituting the dominant coupling mechanism. ${ }^{14}$

Basic analysis shows that the phase between a laser field and the field coupled to it from the adjacent lasers is ${ }^{14}$

$$
\Delta \phi=\left(\pi \zeta n_{\mathrm{erf}} / 2\right)\left(l^{2} / \lambda L_{d}\right),
$$

where $l$ is the center-to-center spacing between two adjacent lasers, $\lambda$ is the (free space) wavelength, $L_{d}$ is the length of the diffraction section of the resonator, $n_{\text {eff }}$ is the effective index of the laser mode, and $\zeta$ is a constant depending on $l, L_{d}$ and the confocal parameter of the resonator $Z_{0},{ }^{15}$ whose values lie between 1 (for $Z_{0} \gg L_{d}$ ) and 2 (for $Z_{0} \ll L_{d}$ ). If $\cos (\Delta \phi)>0$, then the lasers in the array will tend to operate in phase, and the resulting far-field pattern will have a single lobe. On the other hand, if $\cos (\Delta \phi)<0$, the lasers in the array will tend to operate out of phase, with a resulting double-lobed far-field pattern. Thus, by changing $L_{d}$, we can change the far-field pattern of the array. Another advantage of having a diffraction region near the mirrors is the expected reduction of the optical field intensity at the facets.

Fabrication of the array starts with the growth of four layers on an $n^{+}$GaAs substrate by liquid phase epitaxy (LPE) at $800^{\circ} \mathrm{C}$. The typical layer thicknesses are $3 \mu \mathrm{m}$ for the lower $n-\mathrm{Ga}_{0.6} \mathrm{Al}_{0.4}$ As layer, $0.25 \mu \mathrm{m}$ for the undoped GaAs active layer, $2 \mu \mathrm{m}$ for the upper $n-\mathrm{Ga}_{0.6} \mathrm{Al}_{0.4}$ As layer, and $0.4 \mu \mathrm{m}$ for the top $p^{+}$-GaAs contact layer. Next, 3- $\mu \mathrm{m}$ wide and $100-\mu \mathrm{m}$-long lines are defined photolithographically on the wafers with center-to-center spacing of $9 \mu \mathrm{m}$, and periodicity of $250 \mu \mathrm{m}$ in their long dimension. The lines, which are parallel to the $\langle 110\rangle$ direction, are subsequently etched with a $\mathrm{H}_{2} \mathrm{SO}_{4}: \mathrm{H}_{2} \mathrm{O}_{2}: \mathrm{H}_{2} \mathrm{O}(1: 8: 8)$ solution down into the upper cladding layer, to within about $0.5 \mu \mathrm{m}$ to $1 \mu \mathrm{m}$ from the active region. This ensures effective optical isolation between adjacent waveguides. A CrAu contact is evaporated over the whole wafer to form the $p$ contact. Since the contact resistance is much higher in the etched regions, most of the current flows through the $p^{+}$contact layer. Then a $100-\mu \mathrm{m}$-wide mesa, parallel to the etched lines, is formed with the same etchant $(1: 8: 8)$ down to the substrate. The substrate is then lapped and layers of $\mathrm{AuGe}$ and $\mathrm{Au}$ are deposited, followed by alloying at $380^{\circ} \mathrm{C}$. Cleaving of the de-

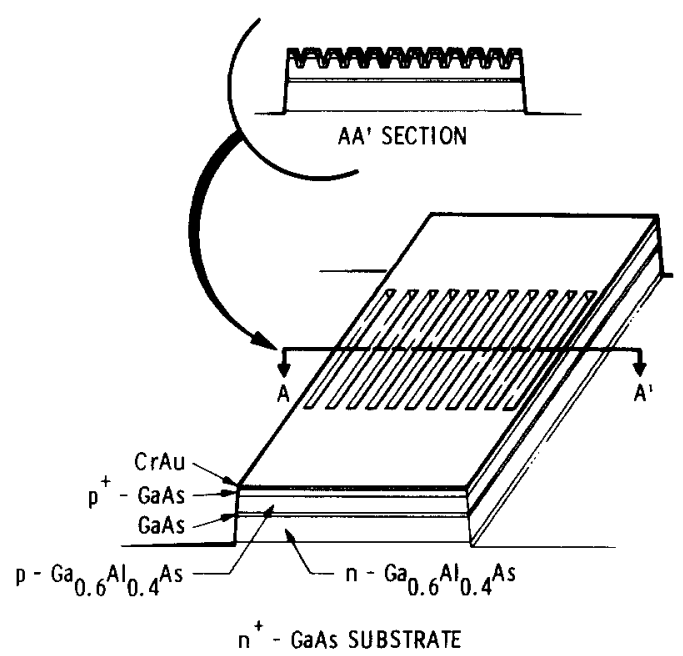

FIG. 1. Schematic diagram of a diffraction coupled phase-locked semiconductor laser array. 

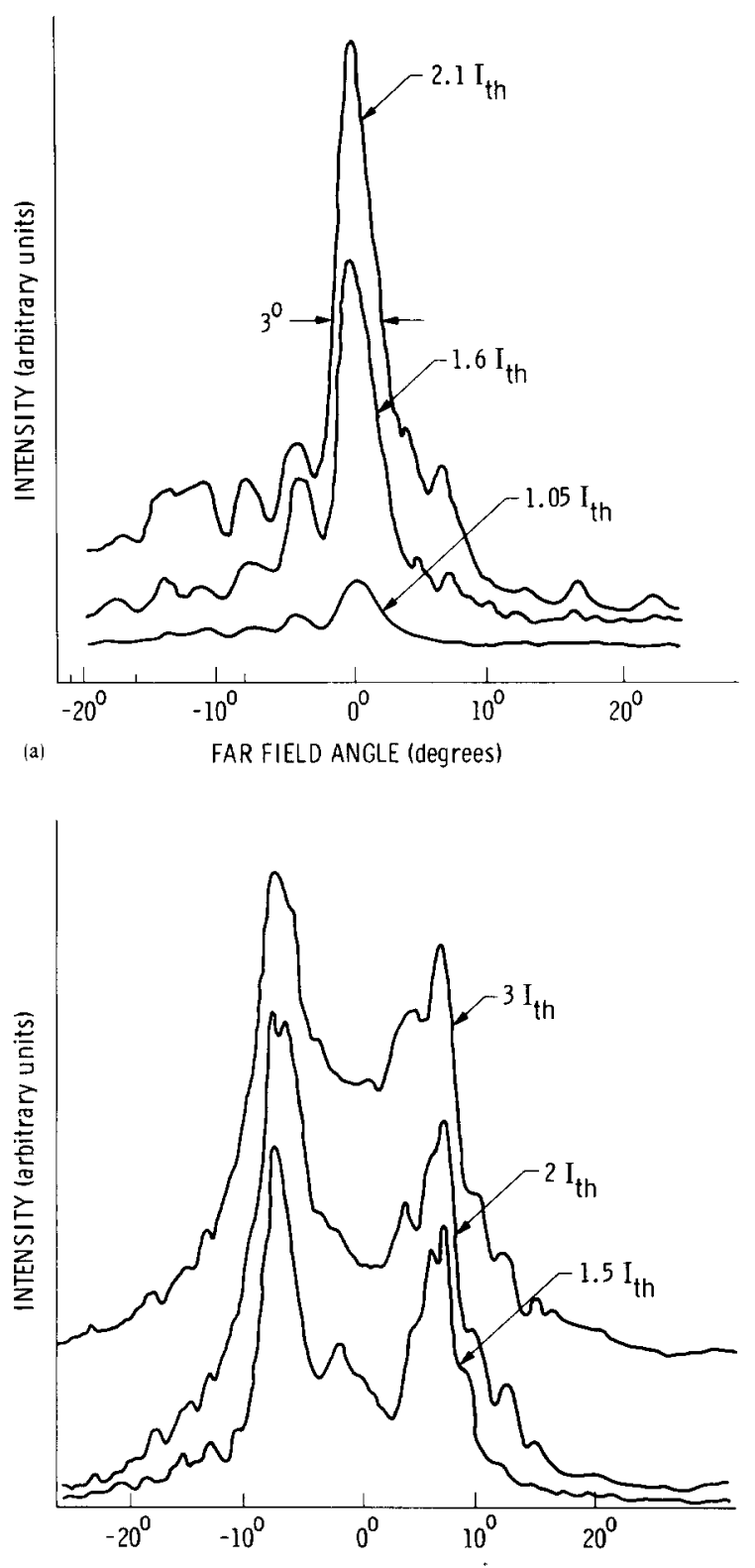

(b)

FAR-FIELD ANGLE (degrees)

FIG. 2. Far-field patterns of diffraction coupled phase-locked semiconductor laser array. (a) Lasers operating in phase, (b) lasers operating out of phase.

vices is done in a direction perpendicular to the etched lines. The cleavage planes lie in the unetched regions. Typical lengths of individual devices are about $250 \mu \mathrm{m}$.

The devices were operated under low duty cycle pulsed conditions. Threshold currents of the arrays were as low as $250 \mathrm{~mA}$, with $400 \mathrm{~mA}$ being a typical value (this corresponds to threshold current densities of $\left.1-1.5 \mathrm{kA} / \mathrm{cm}^{2}\right)$. The differential quantum efficiency is $40 \%$, with linear light versus current characteristics. Peak output powers of up to $1.1 \mathrm{~W}$ were obtained. Although these power levels can be obtained from conventional broad area lasers, in our case the nearfield and far-field patterns are stable over the entire operating region and they do not exhibit random filamentary operation. In addition there is the advantage of the far-field beam narrowing due to the phase locking among the devices.

Shown in Fig. 2 are far-field radiation patterns of the
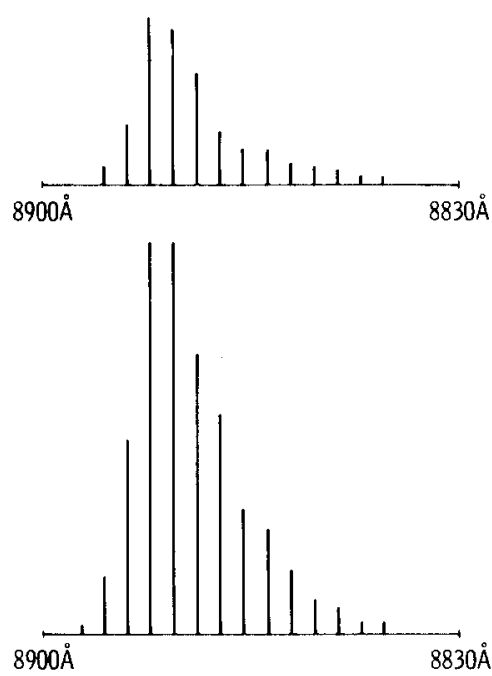

FIG. 3. Spectra of two different lasers in a diffraction coupled phase-locked semiconductors laser array. The laser spacing is $27 \mu \mathrm{m}$.

diffraction couple arrays in the junction plane. As discussed earlier, adjacent lasers in the array can operate in phase or out of phase, depending on the resonator parameters. The inphase operation results in the far-field pattern such as the one shown in Fig. 2(a). The half-beam width is about $3^{\circ}$. A typical far-field pattern for the out of phase case is shown in Fig. 2(b). The separation between the lobes is about $14^{\circ}$ and the width of each lobe is again about $3^{\circ}$. In both cases the radiation patterns change little as the output power levels are increased. The fact that the far-field pattern, although much narrower compared to that of a single laser, is wider than that expected for a diffraction limited uniformly illuminated $100-\mu \mathrm{m}$ aperture is attributed to the fact that due to nonuniformities inherent to the LPE growth process not all the lasers in the array operate at the same level above the threshold. This was confirmed also by the other measurements which are described below. It is expected that employing growth processes such as molecular bear. epitaxy (MBE) or metalorganic chemical vapor deposition (MOCVD), which produce far superior uniformity, ${ }^{16}$ will improve results for the diffraction coupled array, similarly to the improvements achieved in distributed coupling arrays (e.g., Refs. 11 and 10, using MOCVD vs Refs. 1 and 2, respectively, using LPE).

Light spots of individual lasers are observed in the near field of the array since the diffraction section of the resonator is not long enough to completely obliterate the identity of the individual beams. (This happens in the farfield only.) As an additional verification (besides the narrowing of the far-field pattern) that lasers within the array are phase locked, their individual spectra were measured. A typical result is shown in Fig. 3. It is clearly seen that the spectra of the two lasers are identical even though they are located $27 \mu \mathrm{m}$ from one another.

In conclusion, we have demonstrated the operation of a diffraction coupled monolithic semiconductor laser array. This device may be useful in applications where high peak powers and stable radiation patterns are important.

The research described in this paper was carried out by the Jet Propulsion Laboratory, California Institute of Tech- 
nology, under contract with the National Aeronautics and Space Administration. A portion of this research was sponsored by the Office of Naval Research and the National Science Foundation.

'D. R. Scifres, R. D. Burnham, and W. Streifer, Appl. Phys. Lett. 33, 1015 (1978).

${ }^{2}$ D. R. Scifres, W. Streifer, and R. D. Burnham, Appl. Phys. Lett. 34, 259 (1979).

${ }^{3}$ W. T. Tsang, R. A. Logan, and R. P. Salathe, Appl. Phys. Lett. 34, 162 (1979).

${ }^{4}$ D. R. Scifres, W. Streifer, and R. D. Burnham, IEEE J. Quantum Electron. QE-15, 917 (1979).

${ }^{5}$ D. E. Ackley and R. W. H. Engelmann, Appl. Phys. Lett. 39, 27 (1981).

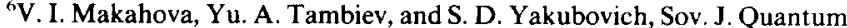
Electron 11, 1351 (1981).

${ }^{7}$ M. B. Small and R. T. Lynch, Jr., J. Appl. Phys. 52, (1981)

${ }^{8}$ I. Suemune, M. Kohno, and M. Yamanishi, Electron. Lett. 18, 745 (1982).

${ }^{9}$ D. R. Scifres, R. D. Burnham, and W. Streifer, Electron. Lett. 18, 549 (1982).

${ }^{10}$ D. R. Scifres, R. D. Burnham, and W. Streifer, Appl. Phys. Lett. 41, 118 (1982).

'D. Scifres, R. D. Burnham, W. Streifer, and M. Burnstein, Appl. Phys. Lett. 41, 614 (1982).

${ }^{12}$ D. E. Ackley, IEEE J. Quantum Electron. QE-18, (1982).

${ }^{13}$ J. E. Ripper and T. L. Paoli, Appl. Phys. Lett. 17, 371 (1970).

14J. Katz (unpublished).

${ }^{15}$ A. Yariv, Quantum Electronics, 2nd ed. (Wiley, New York, 1975), Chap. 6.

${ }^{16}$ R. D. Burnham, D. R. Scifres, and W. Streifer, Appl. Phys. Lett. 40, 118 (1982).

\title{
1-Gbit/s code generator and matched filter using an optical fiber tapped delay line
}

\author{
K. P. Jackson, S. A. Newton, and H. J. Shaw \\ Edward L. Ginzton Laboratory, W. W. Hansen Laboratories of Physics, Stanford University, Stanford, \\ California 94305
}

(Received 6 December 1982; accepted for publication 18 January 1983)

\begin{abstract}
The macrobend optical fiber tapped delay line is a new device which is ideally suited for the processing of signals that have very large bandwidths. Two new applications of this device are presented: code generation and matched filtering. An 8-bit code at $1 \mathrm{Gbit} / \mathrm{s}$ was generated with a programmable eight tap delay line having 1-ns tap intervals. Matched filtering at a rate of $1 \mathrm{Gbit} / \mathrm{s}$ was also demonstrated with a similar deivce. This letter presents the first fiber optic code generator/matched filter of its kind.
\end{abstract}

PACS numbers: $42.80 . \mathrm{Mv}, 06.60 . \mathrm{Jn}$

Code generation and matched filtering can be performed using a variety of approaches. Digital techniques have been used in commercial code generators operating at rates of up to $2 \mathrm{Gbit} / \mathrm{s}$ and state-of-the-art devices empisying GaAs have been demonstrated at $5 \mathrm{Gbit} / \mathrm{s} .{ }^{1}$ Code generation as well as matched filtering can be implemented with surface acoustic wave tapped delay lines ${ }^{2}$ and charge coupled devices. ${ }^{3,4}$ These technologies are largely limited to bandwidths of several hundred megahertz. Magnetostatic wave ${ }^{5}$ and superconducting ${ }^{6}$ tapped delay lines can also be used to perform matched filtering with predicted bandwidths of up to $20 \mathrm{GHz}$. Fiber optic delay lines utilizing single mode fiber $^{7,8}$ are of considerable interest for high-speed signal processing. Because of the inherently large modulation bandwidth and low loss of single mode fiber, these delay lines can have bandwidths of $100 \mathrm{GHz}$ or more.

The macrobend optical fiber tapped delay line is a new device which uses single mode fiber as the delay medium. ${ }^{9}$ It has been previously demonstrated as a frequency filter having a fundamental passband at $1 \mathrm{GHz} .^{9}$ In this letter we report on two new applications of this device: the use of the macrobend optical fiber tapped delay line as a code generator and as a matched filter.

For the applications described in this letter, the macro- bend tapped delay line is configured as shown in Fig. 1. Small amounts of an input signal are tapped out of the fiber by the tapping pin which induces bending losses at regular intervals. The tangentially radiated tap light is collected by the first lens and imaged at an intermediate plane. A transmission mask is inserted at this plane so that individual taps can be weighted by spatial filtering. The second lens then

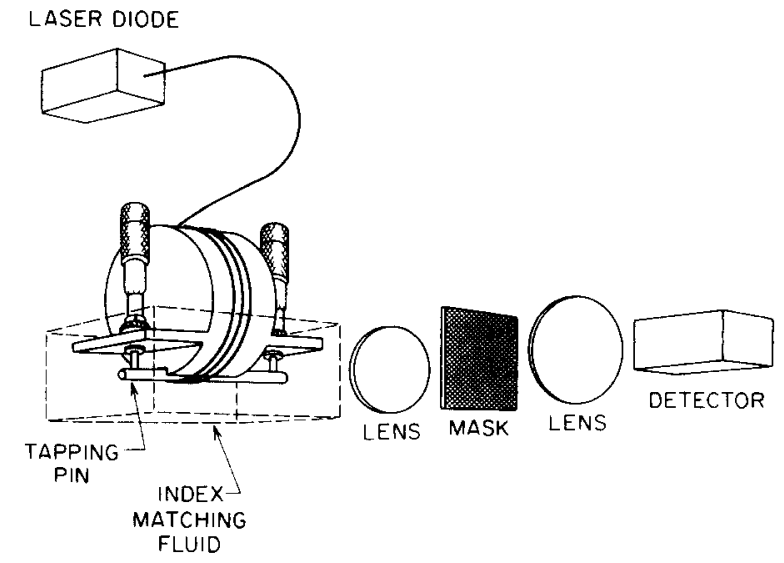

FIG. 1. Schematic diagram of code generator/matched filter using the macrobend optical fiber tapped delay line. 